\title{
Electromodulation spectroscopy of heavy-hole, light-hole, and spin-orbit transitions in GaAsBi layers at hydrostatic pressure
}

\author{
F. Dybała ${ }^{1}$, J. Kopaczek ${ }^{1}$, M. Gladysiewicz ${ }^{1}$, E.-M. Pavelescu ${ }^{2}$, C. Romanitan ${ }^{2}$, \\ O. Ligor ${ }^{2}$, A. Arnoult ${ }^{3}$, C. Fontaine ${ }^{3}$, and R. Kudrawiec ${ }^{1, *}$ \\ ${ }^{1}$ Faculty of Fundamental Problems of Technology, Wroclaw University of Science and \\ Technology, Wybrzeze Wyspianskiego 27, 50-370 Wrocław, Poland \\ ${ }^{2}$ National Institute for Research and Development in Microtechnologies, \\ Erou Iancu Nicolae 126A, 077190, Bucharest, Romania \\ ${ }^{3}$ LAAS, CNRS, University of Toulouse, 7 avenue du Colonel Roche, BP 54200, \\ 31031 Toulouse CEDEX 4, France \\ *Corresponding author e-mail: robert.kudrawiec@pwr.edu.pl
}

GaAsBi layers of various Bi concentrations have been grown by molecular beam epitaxy on GaAs substrate and studied by electromodulation spectroscopy (EM). Optical transitions related to the heavy-hole (HH) and light-hole ( $\mathrm{LH})$ bands as well as the spin-orbit (SO) splitoff band have been observed in EM spectra and their energies have been found to be in very good agreement with theoretical predictions, which take into account the strain-related shifts obtained from the Bir-Pikus theory implemented to the electronic band structure of GaAsBi obtained after recent DFT calculations for this alloy. The pressure coefficients for HH, LH and SO transitions have been determined from PR measurements performed at various hydrostatic pressures and discussed. 
Dilute bismides (III-V alloys with a few percent of Bi atoms) are interesting because of their potential applications in lasers [1-7] and unusual opto-electronic properties like a strong Bi-related increase in the spin-orbit splitting, the band anticrossing interaction in the valence band or carrier localization [8-12]. For recent years GaAsBi was the most intensively explored alloy among dilute bismides [13-20], but many properties of this alloy are still unexplored. Ones of them are pressure coefficients for optical transitions in GaAsBi. The aim of this paper is to study $\mathrm{HH}, \mathrm{LH}$, and SO transitions in GaAsBi by electromodulation (EM) spectroscopy (i.e., photoreflectance (PR) and contactless electroreflectance (CER)) and discuss the influence of $\mathrm{Bi}$ atoms on pressure coefficients observed for these transitions.

EM spectroscopy is known as an excellent method to study the strain-related changes in the electronic band structure [21, 22]. Due to its differential-like character this technique is not sensitive to indirect optical transitions and allows the precise study of direct optical transition energies as well as their shift under the built-in strain, including strain-related splitting between the HH and LH subbands [11, 21, 22, 25]. So far, EM spectroscopy has been applied a few times to study GaAsBi [11, 20-2616-20] but issues of the strain related valence band splitting and the pressure coefficients for direct optical transitions were not intensively explored for this alloy. In this paper, we apply EM spectroscopy to investigate $\mathrm{GaAs}_{1-\mathrm{x}} \mathrm{Bi}_{\mathrm{x}}$ layers with various $\mathrm{Bi}$ concentrations (1.2, 2.2, 3.1, 3.7, and $5.4 \% \mathrm{Bi}$ ) grown by molecular beam epitaxy on GaAs substrate. We have observed that energies of $\mathrm{HH}, \mathrm{LH}$ and $\mathrm{SO}$ transitions are in very good agreement with theoretical predictions, which take into account the strain-related shifts obtained from the Bir-Pikus theory implemented to the electronic band structure of $\mathrm{GaAs}_{1-\mathrm{x}} \mathrm{Bi}_{\mathrm{x}}$ obtained after recent DFT calculations [27, 28] for this alloy, and determined the pressure coefficients for direct optical transitions in $\mathrm{GaAs}_{1-\mathrm{x}} \mathrm{Bi}_{\mathrm{x}}$ by $\mathrm{PR}$ measurements at various hydrostatic pressures. 
GaAsBi layers were grown by molecular beam epitaxy (MBE) on semi-insulating (001) GaAs substrates. After the growth of a GaAs buffer layer at $580^{\circ} \mathrm{C}$, the substrate temperature was ramped down to the temperature used for $\mathrm{GaAsBi}$ growth. The temperature was in range of $390-350^{\circ} \mathrm{C}$ depending on the bismuth content to be incorporated. The arsenic flux was adjusted in order to provide about stoichiometric V/III conditions for a growth rate of 0.3 $\mathrm{ML} / \mathrm{s}$. Once the substrate temperature stabilized, $\mathrm{Bi}$ and $\mathrm{Ga}$ were provided simultaneously [14].

CER and PR measurements have been performed in the so called 'bright configuration' [29, 30]. For PR measurements with hydrostatic pressures the samples were mounted on the plug with a sapphire window inside a pressure cell [29]. The pressure was increased by pushing the piston inwards the cell body. The liquid used as a pressure medium was Daphne 7474 remained hydrostatic and transparent in the whole pressure range (20 kbar). The pressure was determined by changes of the resistance of an InSb sensor which gives about 0.1 kbar sensitivity in the 20 kbar range.

Since bismuth incorporation is known to be highly dependent on substrate temperature, X-ray diffraction was used to accurately determine the bismuth content as well as the degree of strain relaxation in the studied epilayers. Figure 1 shows the XRD $2 \theta-\omega$ scan curve in the 004 reflection for the different GaAsBi samples with various Bi concentrations, recorded by means of a D8 Discover Bruker X-ray Diffraction System. For each diffractogram the strongest peak is associated with the GaAs substrate, whose angle is constant. The peak on its left side corresponds to the GaAsBi layer. Its angular position changes from sample to sample due to the lattice constant of GaAsBi increasing with the Bi concentration. The presence of thickness fringes indicates the high quality of these layers. 224 reciprocal space mapping was used to evaluate the degree of strain relaxation in GaAsBi layers. The strain relaxation for 
GaAsBi layer with the highest Bi concentration is very low and estimated to be of the order of $\sim 10 \%$. For the remaining samples the relaxation degree in $\mathrm{GaAs}_{1-\mathrm{x}} \mathrm{Bi}_{\mathrm{x}}$ layer is close to zero. It means that $\mathrm{GaAs}_{1-\mathrm{x}} \mathrm{Bi}_{\mathrm{x}}$ layers can be treated as fully strained on GaAs while the sample with higher $\mathrm{Bi}$ concentration (5.4 \% Bi) as almost fully strained since its relaxation degree is very low.

The content of $\mathrm{GaAs}_{1-\mathrm{x}} \mathrm{Bi}_{\mathrm{x}}$ layers is determined assuming that the lattice constant of $\operatorname{GaAs}_{1-\mathrm{x}} \mathrm{Bi}_{\mathrm{x}}$ changes linearly with Bi concentration. The in-plane $\left(\varepsilon_{||}\right)$and perpendicular $\left(\varepsilon_{\perp}\right)$ strain in $\mathrm{GaAs}_{1-\mathrm{x}} \mathrm{Bi}_{\mathrm{x}}$ layer are linked through the tetragonal strain relation $\varepsilon_{\perp}=-2 \frac{C_{12}}{C_{11}} \varepsilon_{||}$, where the $C_{11}$ and $C_{12}$ are the elastic constants of $\mathrm{GaAs}_{1-\mathrm{x}} \mathrm{Bi}_{\mathrm{x}}$. The in-plane and perpendicular strain in fully strained $\mathrm{GaAs}_{1-\mathrm{x}} \mathrm{Bi}_{\mathrm{x}}$ layer on GaAs are given by $\varepsilon_{||}=\left(\left(a^{\text {GaAs }}-a^{\text {GaAsBi }}\right) / a^{\text {GaAsBi }}\right)$ and $\varepsilon_{\perp}=\left(\left(a_{\perp}{ }^{G a A s B i}-a^{G a A s B i}\right) / a^{G a A s B i}\right)$ formula, respectively, where the lattice constant $a_{\perp}{ }^{G a A s B i}$ is determined from the $2 \theta-\omega$ scan of the (004) reflection. The elastic constants of $\mathrm{GaAs}_{1-\mathrm{x}} \mathrm{Bi}_{\mathrm{x}}$ layer $\left(C_{11}\right.$ and $\left.C_{12}\right)$ used to determine the Bi concentration from above formulas are calculated using a linear interpolation with values of $C_{11}=1221 \mathrm{GPa}$ and $C_{12}=566 \mathrm{GPa}$ for GaAs [31] and $C_{11}=816 \mathrm{GPa}$ and $C_{12}=281 \mathrm{GPa}$ for $\mathrm{GaBi}$ [32]. The lattice constants of GaAs and GaBi are assumed to be 5.65 and $6.28 \AA[31,32]$.

Figure 2 and 3 show CER and PR spectra, respectively, measured at room temperature in the vicinity of the direct bandgap $\left(E_{0}\right)$ and the spin-orbit split $\left(E_{0}+\Delta_{S O}\right)$ transitions for the five $\mathrm{GaAs}_{1-\mathrm{x}} \mathrm{Bi}_{\mathrm{x}}$ samples of different $\mathrm{Bi}$ concentrations. In the range of bandgap transition two CER (and PR) resonances are clearly visible. Because of the compressive strain in GaAsBi layer the low energy resonance is attributed to the optical transition between the heavy-hole subband and the conduction band (HH transition) while the second resonance is attributed to the optical transition between the light-hole subband and the conduction band (LH transition). 
With increasing $\mathrm{Bi}$ concentration the two transitions shift to lower energy and the energy separation between them increases. The spin-orbit transition (transition labeled in Figs. 2 and 3 as SO transition) also shifts to lower energy with increasing Bi concentration but this shift is about one magnitude weaker. Because of this the SO transition in GaAsBi overlaps with the SO transition in GaAs in PR spectra (the spectral range of SO transition in GaAs is marked by grey bar in Figs. 2 and 3). Such a situation is not present in CER spectra since this technique almost does not probe GaAs buffer, i.e., the main band bending modulation is present at the surface of the sample.

In order to extract energies of the optical transitions from experimental data, CER and PR spectra are fitted by the Aspnes formula [33]

$$
\frac{\Delta R}{R}(E)=\operatorname{Re}\left[\sum_{j=1}^{n} C_{j} e^{i \theta_{j}}\left(\hbar \omega-E_{j}+i \Gamma_{j}\right)^{-m_{j}}\right],
$$

where $\frac{\Delta R}{R}(E)$ is the energy dependence of the CER (and PR) signal, $n$ is the number of spectral functions to be fitted, $\hbar \omega$ is the photon energy of the probe beam, $E_{j}$ is the critical point energy, and $\Gamma_{j}, C_{j}$ and $\theta_{j}$ are the broadening, amplitude and phase angle, respectively. The term $m_{j}$ is assumed to be 2.5 , which corresponds to a band-to-band transition. The fitting curves are plotted in Fig. 2 and 3 by thick grey lines together with the moduli of the individual CER (and PR) resonances (color lines) which are obtained from Eq. (2) with parameters derived from the fit.

$$
\Delta \rho_{j}(E)=\frac{\left|C_{j}\right|}{\left[\left(\hbar \omega-E_{j}\right)^{2}+\Gamma_{j}^{2}\right]^{m_{j}}}
$$


Figure 4 shows energies of the optical transitions ( $\mathrm{HH}, \mathrm{LH}$, and SO transitions) extracted from the fitting procedure (CER - solid points; PR - open points) together with theoretical predictions of energies of $\mathrm{E}_{0}$ and $\mathrm{E}_{0}+\Delta_{\mathrm{SO}}$ transitions (thin solid lines) taken after recent DFT calculations $[27,28]$ and energies of $\mathrm{HH}, \mathrm{LH}$, and $\mathrm{SO}$ transitions in fully strained $\mathrm{GaAs}_{1-\mathrm{x}} \mathrm{Bi}_{\mathrm{x}}$ layers (color lines). These energies are calculated utilizing formulas from the Bir-Pikus theory [34]:

$$
\begin{aligned}
& E_{H H}=E_{H H}(\varepsilon=0)+\delta E_{H}-\frac{1}{2} \delta E_{U} \\
& E_{L H}=E_{L H}(\varepsilon=0)+\delta E_{H}+\frac{1}{2} \delta E_{U}-\frac{1}{2} \frac{\delta E_{U}^{2}}{\Delta_{S O}}+\ldots \\
& E_{S O}=E_{S O}(\varepsilon=0)+\delta E_{H}+\frac{1}{2} \delta E_{U}+\frac{1}{2} \frac{\delta E_{U}^{2}}{\Delta_{S O}}+\ldots
\end{aligned}
$$

where $\Delta_{S O}$ is the spin-orbit splitting in $\mathrm{GaAsBi}, \delta \mathrm{E}_{H}$ and $\delta \mathrm{E}_{U}$ are the components describing changes in the band structure related to the hydrostatic and uniaxial deformations, respectively. These components are calculated using the following formulas: $\delta E_{H}=2 a\left(\frac{C_{11}-C_{12}}{C_{11}}\right) \varepsilon_{||}$and $\delta E_{U}=2 b_{a x}\left(\frac{C_{11}+2 C_{12}}{C_{11}}\right) \varepsilon_{||}$, where $a$ and $b_{a x}$ are the hydrostatic and the uniaxial deformation potentials, respectively, and are assumed to be the same as for GaAs. Comparing theoretical predictions with experimental points, we can conclude that the agreement is very satisfactory, and therefore, hydrostatic and uniaxial deformation potentials typical of GaAs can be applied as a first approximation to describe the strain-related changes in the electronic band structure in $\mathrm{GaAs}_{1-\mathrm{x}} \mathrm{Bi}_{\mathrm{x}}$ alloys with low $\mathrm{Bi}$ concentrations ( $\mathrm{Bi} \leq 5.4 \%$ ). A more accurate approach would be to linearly interpolate the 
deformation potentials between $\mathrm{GaAs}$ and $\mathrm{GaBi}$; however, deformation potentials for $\mathrm{GaBi}$ are not available in the literature.

Figure 4 also clearly show that 8-band $\boldsymbol{k p}$ Hamiltonian with Bi-related shift of the conduction band and the valence bands taken after recent DFT calculations [27] can be used to describe the electronic band structure for this alloy. In our opinion it is a very important conclusion for calculations of the material gain for quantum wells containing GaAsBi etc. [3] since the band anticrossing (BAC) model [35] applied to the valence band [3, 8] includes three Bi-related levels and three interaction terms which values are still not well explored and thereby they are under debate.

The main aim of this paper is to study the pressure dependence of optical transitions in GaAsBi alloys since this issue has been not explored yet by absorption-like techniques. So far the pressure coefficient has been determined only for $\mathrm{GaAs}_{0.986} \mathrm{Bi}_{0.014} / \mathrm{GaAs}$ light emitting diodes [36]. The authors reported that the pressure coefficient for electroluminescence peak from the diode is $11.8 \pm 0.3 \mathrm{meV} / \mathrm{kbar}$ at $80 \mathrm{~K}$. Since the electroluminescence peak is very broad and a contribution of localized emission can be present at this temperature, it is interesting to explore pressure coefficients for band-to-band transitions in GaAsBi alloys (i.e., HH, LH and SO transitions).

Figure 5 shows PR spectra measured at room temperature with various hydrostatic pressures for the $\mathrm{GaAs}_{0.946} \mathrm{Bi}_{0.054}$ layer in the vicinity of bandgap (left panel) and $\mathrm{SO}$ transition (right panel). In addition to $\mathrm{HH}, \mathrm{LH}$ and SO transitions in GaAsBi layer, spectral features associated with the bandgap transition in the GaAs buffer are clearly visible in the PR spectra. With increasing hydrostatic pressure all optical transitions shift to blue. Similar measurements have been performed for the $\mathrm{GaAs}_{0.963} \mathrm{Bi}_{0.037}$ sample and corresponding behavior of $\mathrm{PR}$ spectra was observed for this sample. 
Figure 6 shows energies of optical transitions in $\mathrm{GaAs}_{1-\mathrm{x}} \mathrm{Bi}_{\mathrm{x}}$ layers $(\mathrm{Bi}=3.7$ and $5.4 \%)$ and GaAs buffers extracted from PR spectra by using the Aspnes formula. In this range of applied hydrostatic pressures the pressure dependence of the energy of the optical transitions is linear and therefore experimental points are fitted by a linear function. Values of pressure coefficients determined from linear fits are given at proper lines/experimental points in Fig. 6 (a). It is observed that the extracted pressure coefficients for optical transitions in $\mathrm{GaAs}_{1-\mathrm{x}} \mathrm{Bi}_{\mathrm{x}}$ are smaller than those observed for GaAs buffers, i.e. the reference material in this case. Figure 6 (b) shows the relative changes in the transition energy in $\mathrm{GaAs}_{1-\mathrm{x}} \mathrm{Bi}_{\mathrm{x}}$ alloys and $\mathrm{GaAs}$ buffer obtained experimentally in this work (solid points) together with the linear fit of the relative changes (solid lines) determined for the fundamental transition (HH transition for $\mathrm{GaAs}_{1-\mathrm{x}} \mathrm{Bi}_{\mathrm{x}}$ layers and $\mathrm{E}_{0}$ transition for $\mathrm{GaAs}$ ). This comparison clearly shows that pressure coefficients for $\mathrm{GaAs}_{1-\mathrm{x}} \mathrm{Bi}_{\mathrm{x}}$ alloys decreases with the increase in Bi concentration nonetheless this decrease is small in this range of applied pressures - for $\mathrm{GaAs}_{1-\mathrm{x}} \mathrm{Bi}_{\mathrm{x}}$ with 3.7 and $5.4 \% \mathrm{Bi}$ the pressure coefficient is smaller with $\sim 5$ and $\sim 12 \%$, respectively, than for GaAs. It means that as a first approximation, the hydrostatic deformation potentials of GaAs can be applied to describe the pressure-induced changes in the electronic band structure of $\mathrm{GaAs}_{1-\mathrm{x}} \mathrm{Bi}_{\mathrm{x}}$ alloys with low Bi concentrations - this is a very important conclusion for the design of GaAsBi containing heterostructures for optoelectronic applications. The same conclusion has been derived for the uniaxial deformation potential, which describes the splitting between $\mathrm{HH}$ and LH bands as well as the strain-related shift of the SO split-off band. Moreover it is worth to note that differences between pressure coefficients for $\mathrm{HH}, \mathrm{LH}$, and $\mathrm{SO}$ transitions for a given Bi concentration are very small in comparison to the uncertainty of these coefficients, and therefore they are not discussed. 
On the other hand, $\mathrm{Bi}$ related changes in deformation potentials are detected experimentally in this work and therefore it is interesting to explain the observed changes within the BAC model, which very well describes non-linearity in pressure dependence of optical transitions in Ga(In)NAs [35]. This model has been also applied to dilute bismides [3, 8] but pressure dependencies of optical transitions were not explored within this model.

Figure 7 (a) shows the pressure dependence of bandgap for three materials: $\mathrm{GaAs}_{0.97} \mathrm{Bi}_{0.03}, \mathrm{GaN}_{0.03} \mathrm{As}_{0.97}$ and GaAs (i.e., reference compound for the two previous alloys). These dependencies have been calculated within 14-band (GaAsBi) and 10-band (GaNAs) $\boldsymbol{k} \boldsymbol{p}$ model according to Refs.[3, 37]. For GaAsBi the band anticrossing interaction is present in the valence band [8] while for GaNAs this interaction is present in the conduction band [35] as schematically shown in the sketch in Fig. 7. In this case it is important to note that the energy difference between the conduction band minimum and the resonant nitrogen level $(\mathrm{N})$ for $\mathrm{GaN}_{0.97} \mathrm{As}_{0.03}$ is smaller than the energy difference between the valence band maximum and the resonant bismuth level $(\mathrm{Bi})$ for $\mathrm{GaAs}_{0.97} \mathrm{Bi}_{0.03}$ (this difference is 0.23 vs $0.40 \mathrm{eV}$ ). Moreover it is meaningful to note that the conduction band in GaAs shift stronger with the hydrostatic pressure than the valence band (the hydrostatic potential for the conduction band is $-7.17 \mathrm{eV}$ while for the valence band it is $-1.16 \mathrm{eV}$ [31]). Because of this the nonlinearity in pressure dependencies of bandgap are observed for $\mathrm{GaN}_{0.03} \mathrm{As}_{0.97}$ at lower hydrostatic pressures than for $\mathrm{GaAs}_{0.97} \mathrm{Bi}_{0.03}$. This simple comparison of $\mathrm{GaN}_{0.03} \mathrm{As}_{0.97}$ and $\mathrm{GaAs}_{0.97} \mathrm{Bi}_{0.03}$ alloys within BAC model explain our experimental observation of linear dependence of energies $\mathrm{HH}, \mathrm{LH}$ and $\mathrm{SO}$ transitions in $\mathrm{GaAs}_{1-\mathrm{x}} \mathrm{Bi}_{\mathrm{x}}$ layers in the studied range of hydrostatic pressures and lower values of pressure coefficient for $\mathrm{GaAs}_{1-\mathrm{x}} \mathrm{Bi}_{\mathrm{x}}$ alloys in comparison to the GaAs reference compound. Taking into account chemical trends in pressure coefficients in semiconductor alloys [31, 38], an increase in pressure coefficient should be expected with the 
rise of the lattice constant via replacing $\mathrm{As}$ atoms by $\mathrm{Bi}$ atoms. The opposite behavior observed in this work for GaAsBi can be an evidence of band anticrossing interaction in the valence band. In general the interaction between the bismuth-related resonant states and GaAs states in the valence band can be more complex than within BAC model as suggested by Zhang et al. [39].

In the context of the BAC model, it is worthwhile to comment on the optical transitions between the remaining bands predicted within this model. So far such transitions have been observed for dilute nitrides [35, 40-43] but they have never been clearly observed for dilute bismides. However in recent papers $[17,20]$ the authors have observed some experimental evidences for Bi-related resonant states in the valence band in $\mathrm{GaAs}_{1-\mathrm{x}} \mathrm{Bi}_{\mathrm{x}}$ in the transient reflectivity and electroreflectance measurements. These results suggest a mixing mechanism involving more bands than accounted for in the BAC framework [20]. In our case the spectral range between the $\mathrm{HH} / \mathrm{LH}$ and SO transitions has been also very carefully study by CER and PR however we concluded that the GaAs related signal, which is associated with the buffer layer, is dominating in this spectral range in PR spectra and no optical transitions with a good signal to noise ratio, which could be attributed to GaAsBi, are observed in CER spectra. We believe that further studies of the interaction mechanism in the valence band in this material system is very interesting and the hydrostatic pressure applied in this work (i.e., the determined pressure coefficients for $\mathrm{HH}, \mathrm{LH}$ and $\mathrm{SO}$ transitions) throws more light on this matter.

In summary, CER and PR spectroscopy has been applied to study energies of $\mathrm{HH}$, LH, and $\mathrm{SO}$ transitions in compressive strained $\mathrm{GaAs}_{1-\mathrm{x}} \mathrm{Bi}_{\mathrm{x}}$ layers of various $\mathrm{Bi}$ concentrations in the regime of quasi totally elastically strained GaAsBi. It has been found that the strain related shifts of $\mathrm{HH}, \mathrm{LH}$, and SO transition are very well described within Bir-Pikus theory, which is 
consistent with previous studies [11, 25]. In addition pressure coefficients for these transitions have been determined from PR measurements at high hydrostatic pressures. For $\mathrm{GaAs}_{1-\mathrm{x}} \mathrm{Bi}_{\mathrm{x}}$ with 3.7 and 5.4\% Bi these coefficients have been found to be $~ 5$ and $\sim 12 \%$ smaller than for GaAs. It has been concluded that hydrostatic and uniaxial deformation potentials typical of GaAs can be applied as a first approximation to describe the strain-related changes in the electronic band structure in $\mathrm{GaAs}_{1-\mathrm{x}} \mathrm{Bi}_{\mathrm{x}}$ alloys with $\mathrm{Bi}$ concentrations $\leq 5.4 \%$.

\section{ACKONOWLEDGMENTS}

The authors acknowledge financial support from the National Science Centre (NSC) Poland (grant no. 2012/07/E/ST3/01742). In addition, F.D. acknowledges for support within the grant FUGA no. 2014/12/S/ST3/00313 from NSC. E.M.P., C.R. and O.L. acknowledge the financial support by a grant of the Romanian National Authority for Scientific Research and Innovation, CNCS - UEFISCDI, project number PN-II-RU-TE-2014-4-2690, and A.A. and C.F. acknowledge the support of the LAAS technology platform, a member of the French CNRS RENATECH network. 


\section{References}

[1] P. Ludewig, N. Knaub, N. Hossain, S. Reinhard, L. Nattermann, I. P. Marko, S. R. Jin, K. Hild, S. Chatterjee, W. Stolz, S. J. Sweeney, and K. Volz, Appl. Phys. Lett. 102, 242115 (2013).

[2] S. J. Sweeney and S. R. Jin, J. Appl. Phys. 113, 043110 (2013).

[3] M. Gladysiewicz, R. Kudrawiec, and M. S. Wartak, J. Appl. Phys. 118, 055702 (2015).

[4] M. Gladysiewicz, R. Kudrawiec, and M. S. Wartak, J. Appl. Phys. 119, 075701 (2016).

[5] I.P. Marko, Ch. A. Broderick, S. Jin, P. Ludewig, W. Stolz, K. Volz, J. M. Rorison, E.P. O’Reilly, and S. J. Sweeney, Scientific Reports 6, 28863 (2016).

[6] O. Delorme, L. Cerutti, E. Luna, G. Narcy, A. Trampert, E. Tournie, and J. B. Rodriguez, Appl. Phys. Lett. 110, 222106 (2017).

[7] B. Fluegel, S. Francoeur, A. Mascarenhas, S. Tixier, E. C. Young, and T. Tiedje, Phys. Rev. Lett. 97, 067205 (2006).

[8] K. Alberi, W. Walukiewicz, K. M. Yu, O. D. Dubon, K. Bertulis, A. Krotkus, Appl. Phys. Lett. 91, 051909 (2007).

[9] R. Kudrawiec, M. Syperek, P. Poloczek, J. Misiewicz, R. H. Mari, M. Shafi, M. Henini, Y. Galvão Gobato, S. V. Novikov, J. Ibáñez, M. Schmidbauer, and S. I. Molina, J. Appl. Phys. 106, 023518 (2009).

[10] C. A. Broderick, M. Usman, S. J. Sweeney and E. P. O’Reilly, Semicond. Sci. Technol. 27, 094011 (2012).

[11] M. Usman, Ch. A. Broderick, Z. Batool, K. Hild, T. J. C. Hosea, S. J. Sweeney, and E. P. O’Reilly, Phys. Rev. B 87, 115104 (2013).

[12] J. Kopaczek, W. M. Linhart, M. Baranowski, R. D. Richards, F. Bastiman, J. P. R. David, and R. Kudrawiec, Semicond. Sci. Technol. 30, 094005 (2015). 
[13] S. Tixier, M. Adamcyk, T. Tiedje, S. Francoeur, A. Mascarenhas, P. Wei, and F. Schiettekatte, Appl. Phys. Lett. 82, 2245 (2003).

[14] H. Makhloufi, P. Boonpeng, S. Mazzucato, J. Nicolai, A. Arnoult, T. Hungria, G. Lacoste, C. Gatel, A. Ponchet, H. Carrere, X. Marie, C. Fontaine, Nanoscale Research Letters 9, 123 (2014).

[15] K. Forghani, A. Anand, L. J. Mawst, T. F. Kuech, J. Cryst. Growth 380, 23-27 (2013).

[16] P. Ludewig, Z.L. Bushell, b, L. Nattermann, N. Knaub, W. Stolz, and K. Volz, J. Cryst. Growth 396, 95-99 (2014).

[17] R. S. Joshya, A. J. Ptak, R. France, A. Mascarenhas, and R. N. Kini, Phys. Rev. B 90, 165203 (2014).

[18] M. Wu, M. Hanke, E. Luna, J. Puustinen, M. Guina, and A. Trampert, Nanotechnology 26, 425701 (2015).

[19] V. Bahrami-Yekta, T. Tiedje, and M. Masnadi-Shirazi, Semicond. Sci. Technol. 30, 094007 (2015).

[20] K. Alberi, D. A. Beaton, and A. Mascarenhas, Phys. Rev. B 92, 241201(R) (2015).

[21] J. Ibanez, R. Kudrawiec, J. Misiewicz, M. Schmidbauer, M. Henini, and M. Hopkinson, J. Appl. Phys. 100, 093522 (2006).

[22] K. Zelazna, M. P. Polak, P. Scharoch, J. Serafinczuk, M. Gladysiewicz, J. Misiewicz, J. Dekoster, R. Kudrawiec, Appl. Phys. Lett. 106, 142102 (2015).

[23] J. Yoshida, T. Kita, O. Wada, and K. Oe, Jap. J. Appl. Phys. Part 1 42, 371-374 (2003).

[24] S. Francoeur, M.-J. Seong, and A. Mascarenhas, S. Tixier, M. Adamcyk, and T. Tiedje, Appl. Phys. Lett. 82, 3874 (2003). 
[25] Z. Batool, K. Hild, T. J. C. Hosea, X. Lu, T. Tiedje, and S. J. Sweeney, J. Appl. Phys. 111, 113108 (2012).

[26] H. Fitouri, Y. Essouda, I. Zaied, A. Rebey, B. El Jani, Optical Materials 42, 67-71 (2015).

[27] M. Polak, P. Sharoch, and R. Kudrawiec, Semicond. Sci. Technol. 30, 094001 (2015).

[28] R. Kudrawiec, J. Kopaczek, M. P. Polak, P. Scharoch, M. Gladysiewicz, J. Misiewicz, R. D. Richards, F. Bastiman, and J. P. R. David, J. Appl. Phys. 116, 233508 (2014).

[29] R. Kudrawiec and J. Misiewicz, Appl. Surf. Sci. 253, 80 (2006).

[30] R. Kudrawiec, Phys. Status Solidi B 247, 1616 (2010).

[31] I. Vurgaftman, J. R. Meyer, and L. R. Ram-Mohan, J. Appl. Phys. 89, 5815 (2001).

[32] M. Ferhat and A. Zaoui, Phys. Rev. B 73, 115107 (2006).

[33] D. E. Aspnes, Surf. Sci. 37, 418 (1973).

[34] G. L. Bir, G. E. Pikus, Symmetry and strain-induced effects in semiconductors, Wiley, New York, 1976.

[35] W. Shan, W. Walukiewicz, J. W. Ager, E. E. Haller, J. F. Geisz, D. F. Friedman, J. M. Olson, and S. R. Kurtz, Phys. Rev. Lett. 82, 1221 (1999).

[36] N. Hossain, I. P. Marko, S. R. Jin, K. Hild, S. J. Sweeney, R. B. Lewis, D. A. Beaton, and T. Tiedje, Appl. Phys. Lett. 100, 051105 (2012).

[37] M. Gładysiewicz, R. Kudrawiec, M. S. Wartak, J. Appl. Phys. 115, 033515 (2014).

[38] S.H. Wei and A. Zunger, Phys. Rev. B 60, 5404 (1999). 
[39] Y. Zhang and A. Mascarenhas, Phys. Rev. B 71, 155201 (2005).

[40] A. Grau, T. Passow, and M. Hetterich, Appl. Phys. Lett. 89, 202105 (2006).

[41] S. Turcotte, S. Larouche, J.-N. Beaudry, L. Martinu, R. A. Masut, P. Desjardins, and R. Leonelli, Phys. Rev. B 80, 085203 (2009).

[42] R. Kudrawiec, A. V. Luce, M. Gladysiewicz, M. Ting, Y. J. Kuang, C. W. Tu, O. D. Dubon, K. M. Yu, and W. Walukiewicz, Phys. Rev. Appl. 1, 034007 (2014).

[43] R. Kudrawiec, P. Sitarek, M. Gladysiewicz, J. Misiewicz, Y. He, Y. Jin, G. Vardar, A.M. Mintarov, J.L. Merz, R.S. Goldman, K.-M. Yu, W. Walukiewicz, Thin Solid Films 567, 101 (2014). 


\section{Figure captions:}

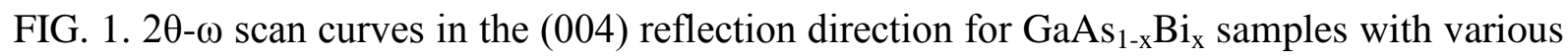
Bi concentrations.

FIG. 2. CER spectra measured at room temperature in the vicinity of the direct bandgap ( $\left.\mathrm{E}_{0}\right)$ and the spin-orbit split $\left(\mathrm{E}_{0}+\Delta_{\mathrm{SO}}\right)$ transitions for the five $\mathrm{GaAs}_{1-\mathrm{x}} \mathrm{Bi}_{\mathrm{x}}$ samples of different $\mathrm{Bi}$ concentrations.

FIG. 3. PR spectra measured at room temperature in the vicinity of the direct bandgap ( $\left.\mathrm{E}_{0}\right)$ and the spin-orbit split $\left(\mathrm{E}_{0}+\Delta_{\mathrm{SO}}\right)$ transitions for the five $\mathrm{GaAs}_{1-\mathrm{x}} \mathrm{Bi}_{\mathrm{x}}$ samples of different $\mathrm{Bi}$ concentrations.

FIG. 4. Energies of the optical transitions (HH, LH, and SO transitions) extracted from CER (solid points) and PR (open points) measurements together with theoretical predictions of energies of $E_{0}$ and $E_{0}+\Delta_{\text {So }}$ transitions (thin black lines), taken after recent DFT calculations [19], and energies of $\mathrm{HH}, \mathrm{LH}$, and $\mathrm{SO}$ transitions in fully strained $\mathrm{GaAs}_{1-\mathrm{x}} \mathrm{Bi}_{\mathrm{x}}$ layers (color lines).

FIG. 5. PR spectra measured at room temperature at various hydrostatic pressures for the $\mathrm{GaAs}_{0.946} \mathrm{Bi}_{0.054}$ layer in the vicinity of bandgap (left panel) and SO transition (right panel).

FIG. 6. (a) Energies of optical transitions in $\mathrm{GaAs}_{1-\mathrm{x}} \mathrm{Bi}_{\mathrm{x}}$ layers $(\mathrm{Bi}=3.7$ and $5.4 \%)$ and $\mathrm{GaAs}$ buffers obtained from PR spectra together with the linear fits and pressure coefficients extracted from these fits. (b) Relative changes in energies of optical transitions in $\mathrm{GaAs}_{1-\mathrm{x}} \mathrm{Bi}_{\mathrm{x}}$ layers $(\mathrm{Bi}=3.7$ and 5.4\%) and GaAs buffers.

FIG. 7. Sketch of band anticrossing interaction in the valence band (GaAsBi case) and in the conduction band (GaNAs case) without and with an applied hydrostatic pressure. (a) Pressure 
dependence of band gap in $\mathrm{GaAs}_{0.97} \mathrm{Bi}_{0.03}, \mathrm{GaN}_{0.03} \mathrm{As}_{0.97}$, and $\mathrm{GaAs}$ host calculated within 14-, 10-, and 8-band $k p$ model, respectively. (b) Comparison of experimental data obtained in this work with theoretical predictions obtained within BAC model for $\mathrm{GaAs}_{0.946} \mathrm{Bi}_{0.054}$. 


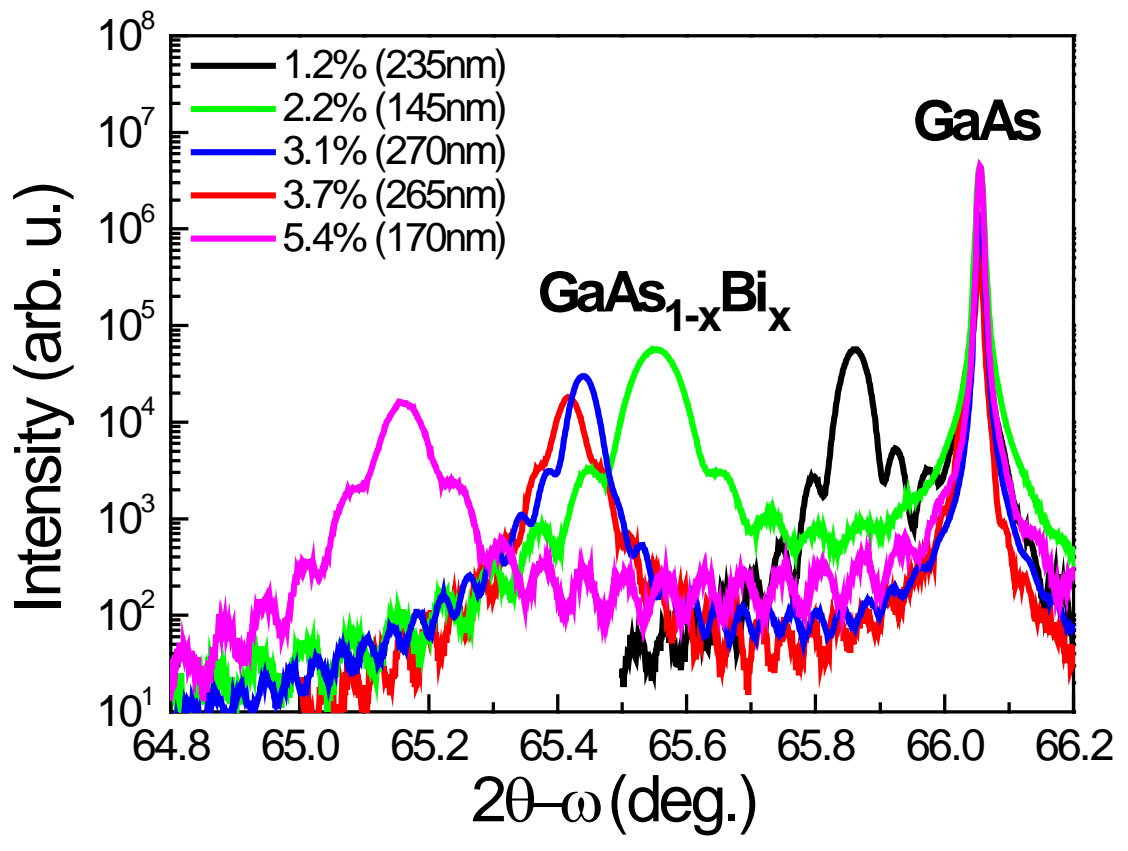

FIG. 1.

(F. Dybala, et al.) 

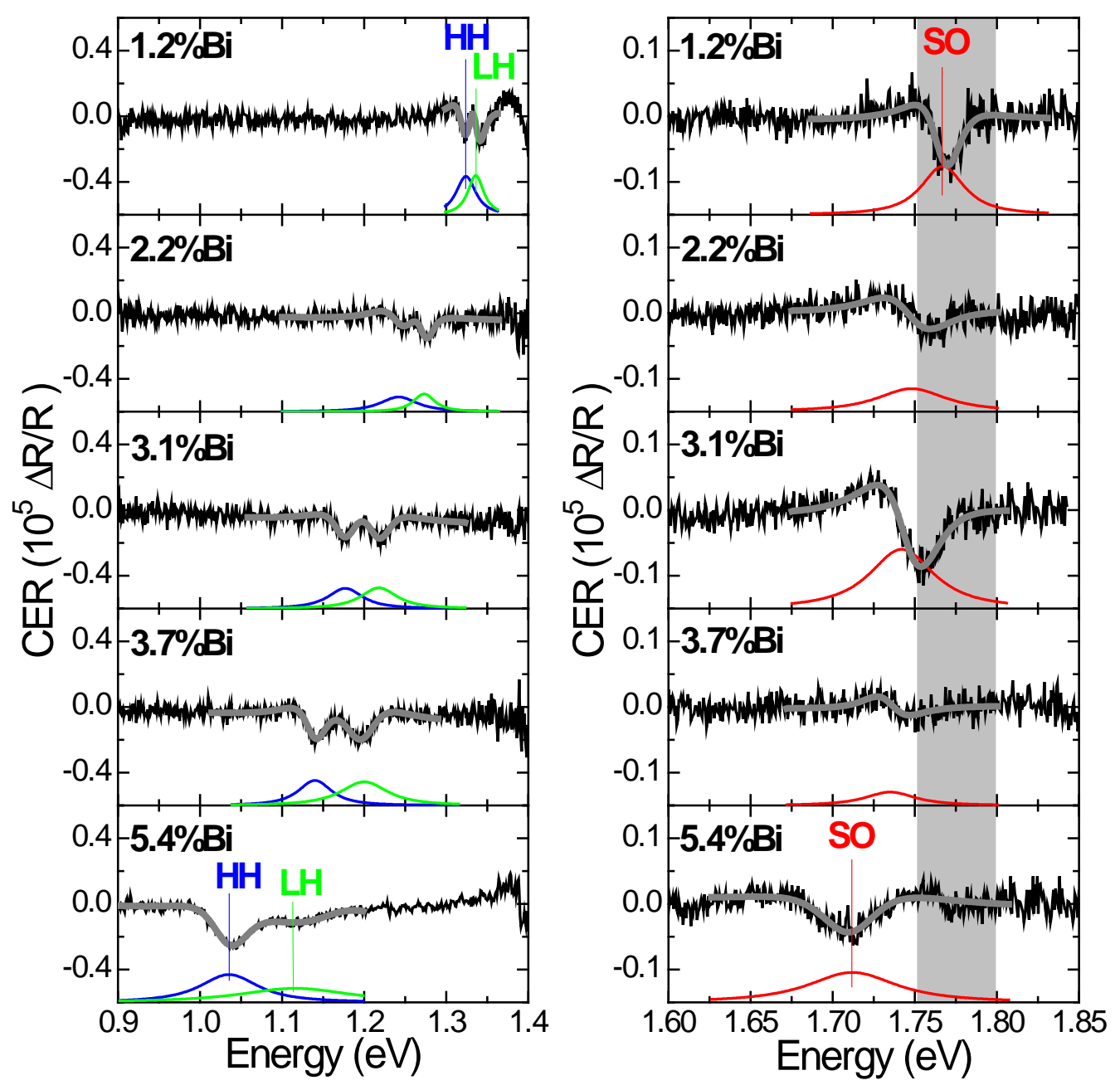

FIG. 2.

(F. Dybala, et al.) 

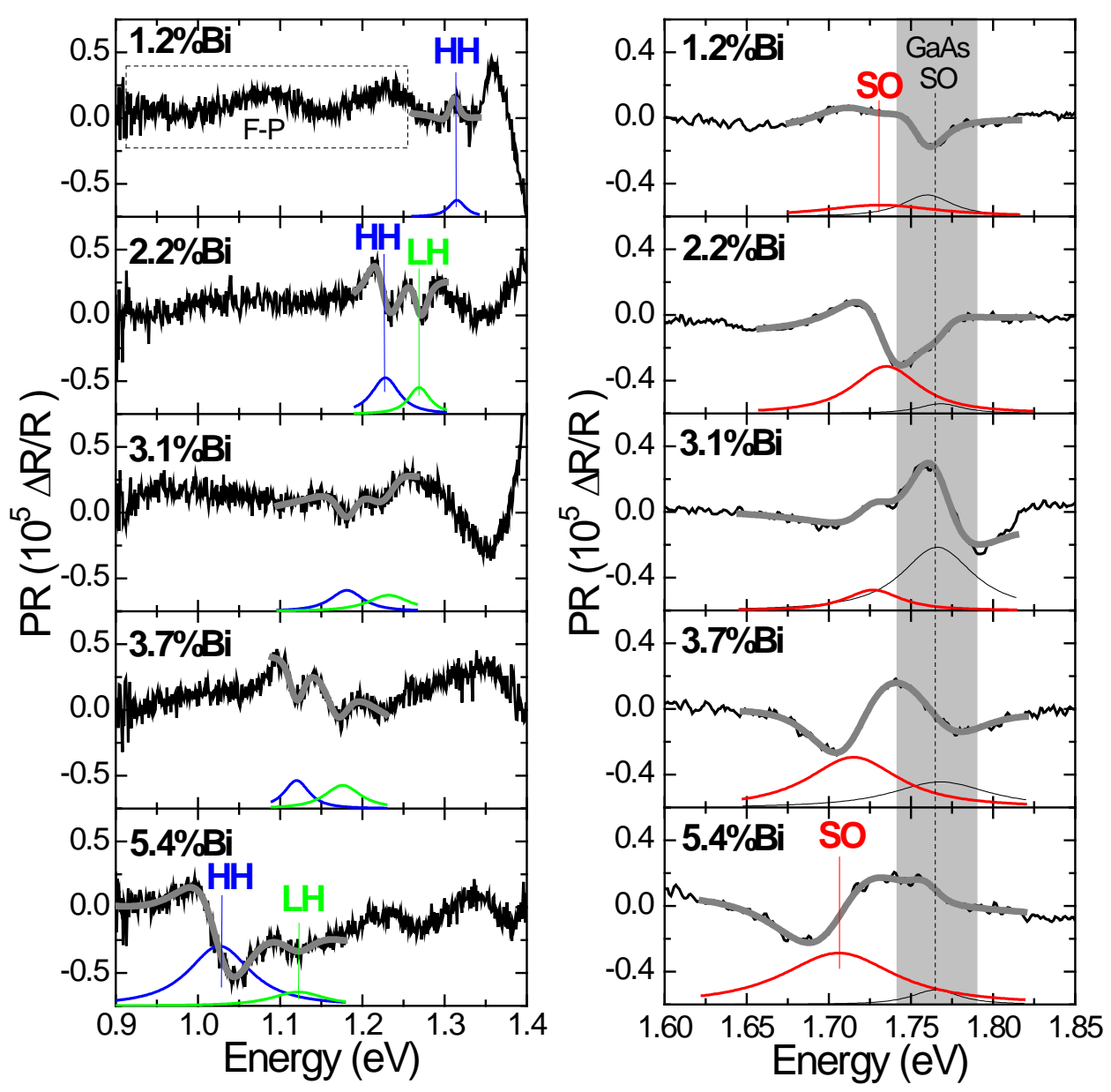

FIG. 3.

(F. Dybala, et al.) 


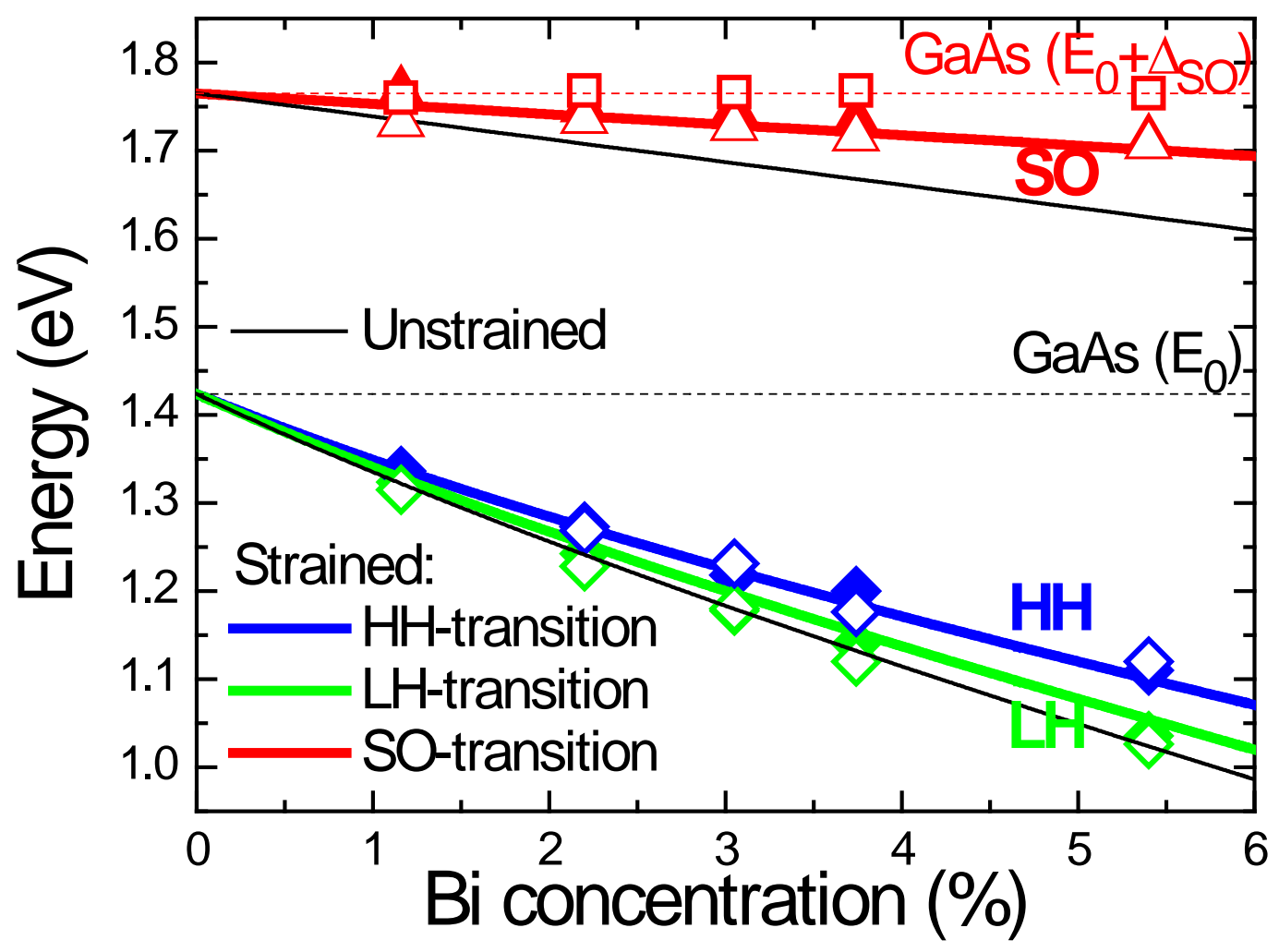

FIG. 4.

(F. Dybala, et al.) 

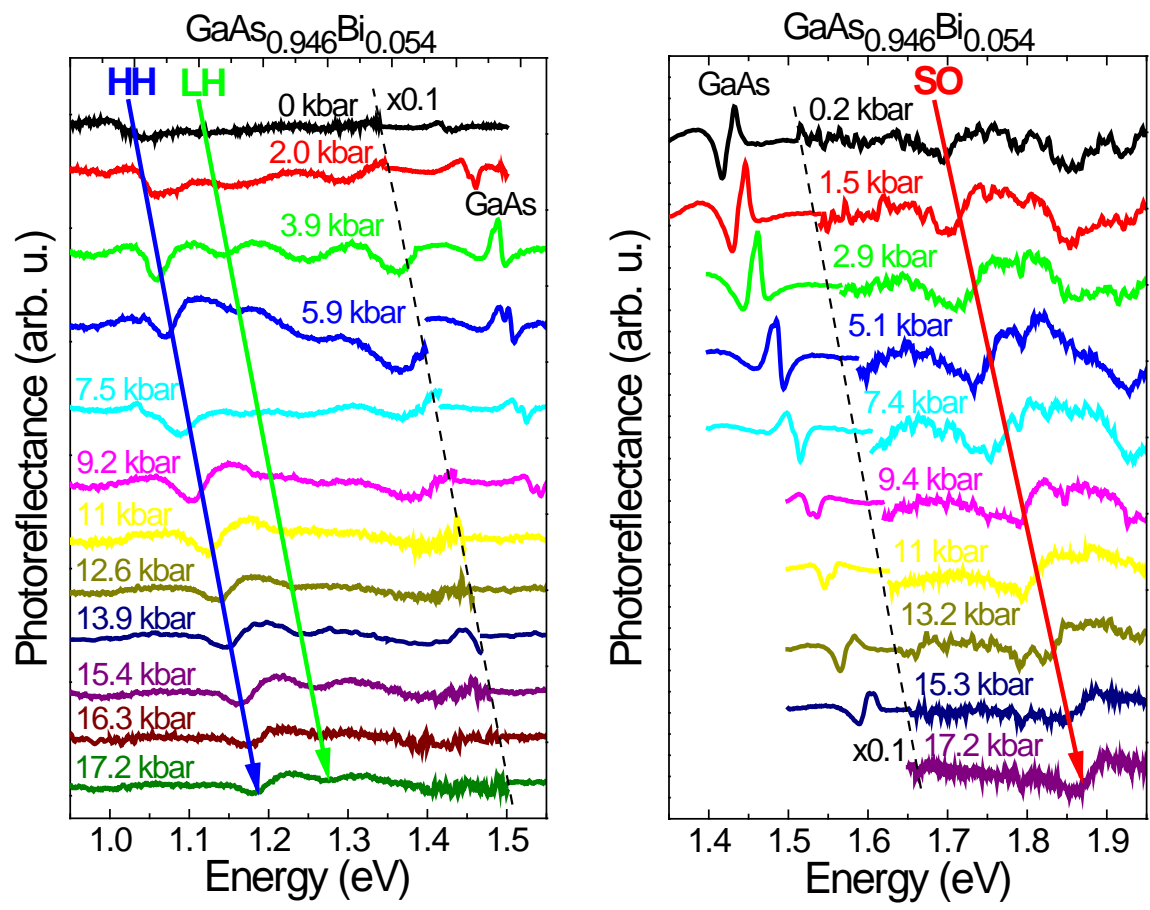

FIG. 5.

(F. Dybala, et al.) 

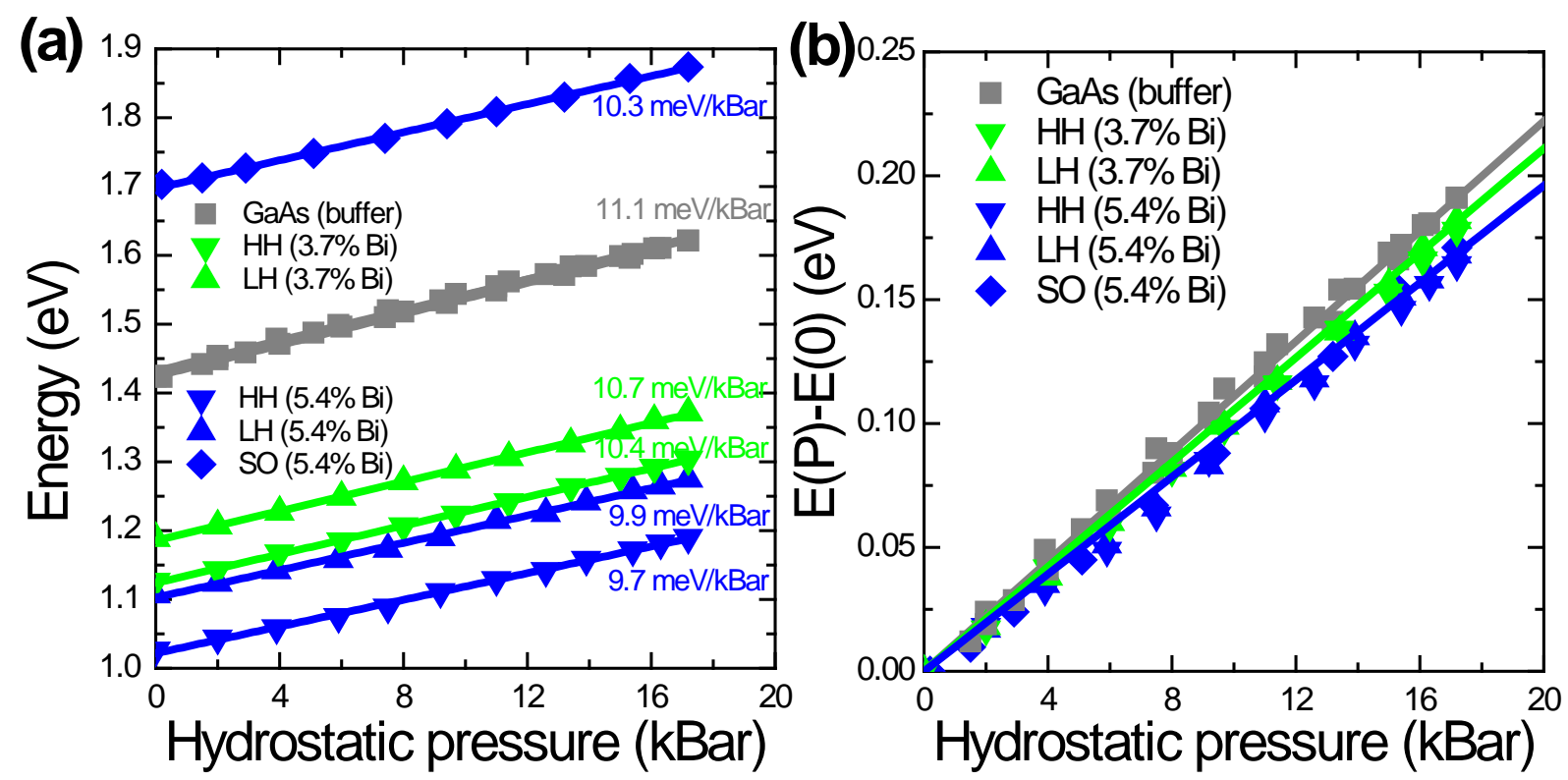

FIG. 6.

(F. Dybala, et al.) 

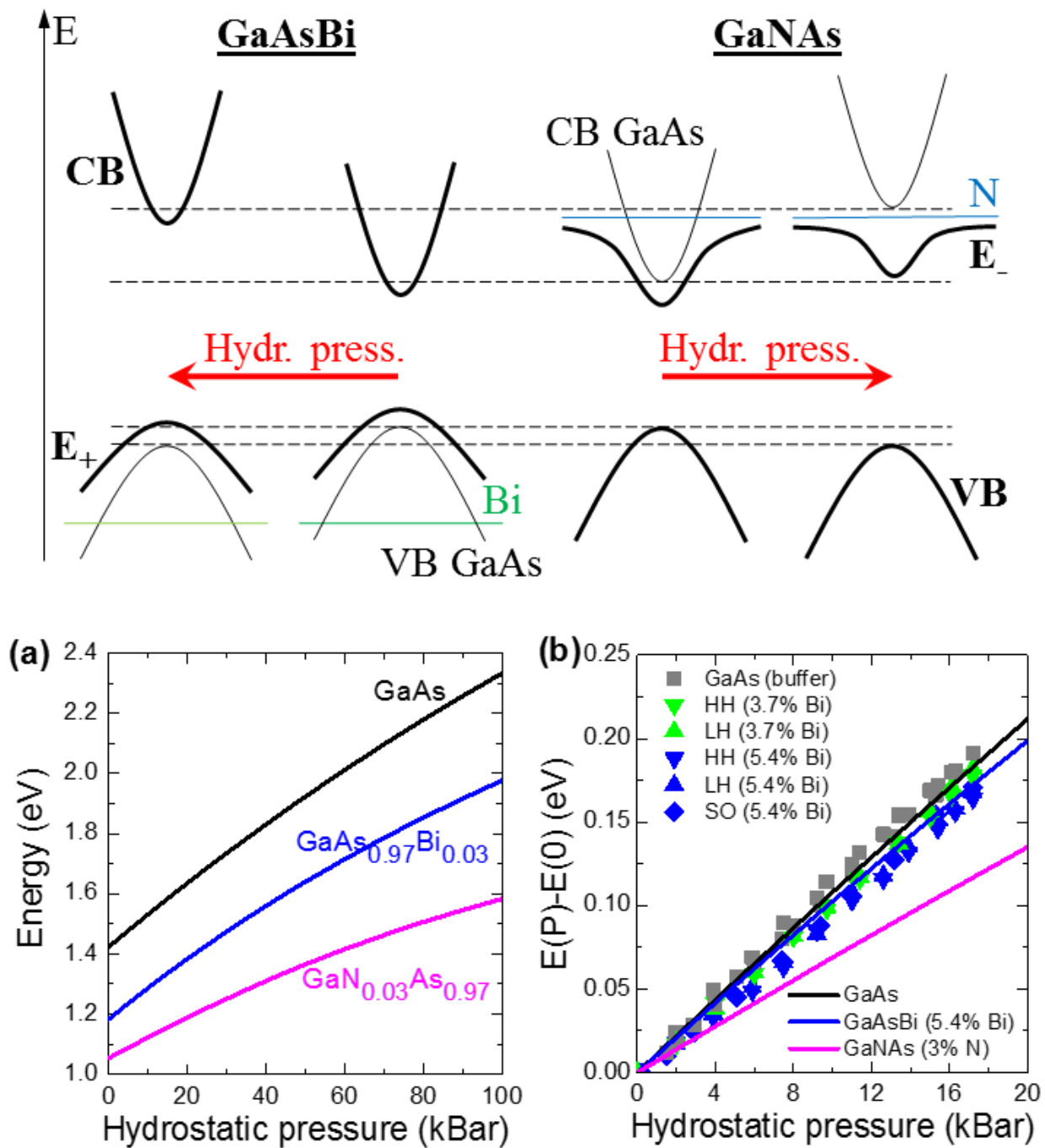

FIG. 7.

(F. Dybala, et al.) 